\title{
Identification of functional information subgraphs in cultured neural networks
}

\author{
Vadas Gintautas*1, Luis MA Bettencourt ${ }^{1,2}$ and Michael I Ham ${ }^{1,3}$
}

Address: ${ }^{1}$ Center for Nonlinear Studies and T-5, Los Alamos National Laboratory, Los Alamos, NM 87545, USA, ${ }^{2}$ Santa Fe Institute, Santa Fe, NM 87501, USA and ${ }^{3}$ Center for Network Neuroscience, University of North Texas, Denton, TX 76203, USA

Email: Vadas Gintautas* - vadasg@lanl.gov

* Corresponding author

from Eighteenth Annual Computational Neuroscience Meeting: CNS*2009

Berlin, Germany. 18-23 July 2009

Published: 13 July 2009

BMC Neuroscience 2009, I0(Suppl I):OI2 doi:10.I I86/I47|-2202-10-SI-OI2

This abstract is available from: http://www.biomedcentral.com//47I-2202//0/SI/OI2

(c) 2009 Gintautas et al; licensee BioMed Central Ltd.

We present a general information theoretic approach for identifying functional subgraphs in complex neuronal networks where the spiking dynamics of a subset of nodes (neurons) are observable. We show that the uncertainty in the state of each node can be written as a sum of information quantities involving a growing number of variables at other nodes. We demonstrate that each term in this sum is generated by successively conditioning mutual information on new measured variables, in a way analogous to a discrete differential calculus. The analogy to a Taylor series suggests efficient optimization algorithms for determining the state of a target variable in terms of functional groups of other nodes. We apply this methodology to electrophysiological recordings of cortical neuronal network grown in vitro. Despite strong stochasticity, we show that each cell's firing is generally explained by the activity of a small number of other neurons. We identify these neuronal subgraphs in terms of their redundant or synergetic character and reconstruct neuronal circuits that account for the state of target cells.

\section{Acknowledgements}

We thank G. W. Gross for sharing his extensive expertise with growing and recording from neuronal cultures. We also thank J. Crutchfield, A. Gutfriend, and A. Hagberg for helpful discussions. This work is supported by LANL's LDRD project 200504IIIER. LA-UR-08-269I. 